\title{
Addressing opioid misuse: Hero Help as a recovery and behavioural health response
}

\author{
Ellen A. Donnelly, Madeline Stenger, ${ }^{\star}$ Shannon Streisel, ${ }^{*}$ Daniel J. O'Connell, ${ }^{\star}$ and Jessica Arnold*
}

This article is related directly to the $6^{\text {th }}$ International Law Enforcement \& Public Health (LEPH) Virtual Conference in March 2021.

\begin{abstract}
Increases in opioid-related overdoses have required law enforcement and public health officials to collectively develop new approaches that treat substance use disorders and save lives. This essay describes the Hero Help recovery and behavioural health assistance program, a Delaware-based initiative providing drug treatment to qualifying adults who contact the police and ask for treatment, or to individuals in lieu of an arrest or upon recommendation by a police officer. Led by the New Castle County Division of Police, this collaborative project has brought together stakeholders from public health and criminal justice to coordinate treatment for people suffering from a substance use disorder and/or mental health problems. This essay describes the goals, evolution, and key activities of the program. It further highlights lessons learned, including improving credibility through concerted community outreach, finding ways to overcome the stigma associated with participating in a law enforcement-based program, gaining officer buy-in, and using data to inform treatment responses. Effectively, this essay seeks to disseminate emerging lessons in creating programming responsive to substance use disorder and mental illness among police departments and their community partners.
\end{abstract}

Key Words Treatment; diversion; mental health; substance misuse

\section{INTRODUCTION}

In the United States, overdoses involving opioids have emerged as a pressing public health problem (Jalal et al., 2018). Fatal drug poisonings have grown fourfold over the last two decades (Hedegaard et al., 2018). Non-fatal overdoses have surged by upwards of $32 \%$ in 16 states within a single year (Vivolo-Kantor, 2018) and frequently occur as a result of polydrug combinations involving opioids with cocaine, amphetamines, or benzodiazepines (Compton et al., 2021; Liu et al., 2020). More recently, the COVID-19 pandemic has accelerated overdose death rates (Stephenson, 2021).

The current opioid crisis has transformed the way that many law enforcement agencies approach enforcing drug laws and assisting people struggling with a substance use disorder (Anderson et al. 2021; Davis et al., 2015; Green et al., 2013; Purviance et al., 2017). Police officers are often the first to arrive at the scene of a potential overdose (Rando et al., 2015), especially in rural areas where emergency medical services may be far away or already in use. More police departments are training their officers to administer naloxone, which reverses the effects of an overdose (Purviance et al., 2017; K. D. Wagner et al., 2015). Increasingly, law enforcement agencies are developing alternatives to formal criminal processing to assist citizens and communities impacted by opioid misuse (PAARI, 2019; Pearlman, 2017). Police departments are beginning to adopt programs that offer referrals to treatment providers, coordinate care, and conduct outreach among overdose survivors and their personal networks (Formica et al., 2018).

This study describes a law enforcement-based treatment program created by the New Castle County Division of Police (abbreviated as NCCPD) in Delaware, in the United States. Known as Hero Help, the project has brought together public health officials, prosecutors, police officers, and community health organizations in hopes of connecting eligible adults to drug and/or alcohol treatment. Beginning as a treatmentreferral effort, Hero Help has grown into a comprehensive care program currently featuring over 500 participants, 52 treatment providers, 5 civilian staff members, and 372 uniformed officers who can serve as program engagers. This essay describes Hero Help's goals and key activities. An

Correspondence to: Ellen A. Donnelly, Center for Drug and Health Studies, University of Delaware, 257 E Main St, Newark, DE 19711, USA. E-mail: done@udel.edu. To cite: Donnelly, E. A., Stenger, M., Streisel, S., O'Connell, D. J., \& Arnold, J. (2021). Addressing opioid misuse: Hero Help as a recovery and behavioural health response. Journal of Community Safety and Well-Being, 6(3), 142-147. https://doi.org/10.35502/jcswb.191

@ Author(s) 2021. Open Access. This work is distributed under the Creative Commons BY-NC-ND license. For commercial re-use, please contact sales@sgpublishing.ca. Sg PUBLISHING Published by SG Publishing Inc. CSKA Official publication of the Community Safety Knowledge Alliance. 
emphasis is placed on the evolution of the program over its five-year history, which may inspire the design of criminal justice and public health responses to opioid misuse in other communities.

\section{PROGRAM CONTEXT}

Communities in New Castle County, Delaware have had to confront their own opioid crisis. At present, Delaware has the second-highest overdose death rate in the United States, trailing West Virginia (Center for Disease Control Injury Center, 2020). Nearly three-quarters of these overdose deaths involve opioids (J. Wagner et al., 2019). NCCPD has jurisdiction over all unincorporated towns and neighbourhoods outside of the city of Wilmington. While serving a non-urban area, the department sees 79\% of Delaware's opioid-related overdoses. Overdose rates have continued to grow here in recent years. For instance, in 2018, NCCPD responded to 571 overdose incidents, with 75 resulting in death. More troubling, these incidents featured repeated locations $(n=194)$ and individuals $(n=174)$.

The urgency of addressing overdoses prompted NCCPD to take action. Started in May 2016, the Hero Help program helps link anyone struggling with substance use issues to immediate treatment services. Qualified individualsDelaware residents aged 18 or older without criminal convictions for serious or violent offences or major criminal charges-may join voluntarily, enter in lieu of arrest, or participate upon recommendation by an officer.

The Hero Help program was initially modelled after the proactive approaches of the Gloucester, Massachusetts, Police Department. Rather than arresting people for drug-related offences, Gloucester's "Angel Initiative" allowed people to visit the police department and ask to be connected to drug treatment without facing criminal prosecution (Pearlman, 2017; Samuels, 2016). The approach relied on volunteers, also known as "angels," to stay with program participants until officers completed the intake, found an open bed, and organized transport (Gloucester Police Department, 2015).

Hero Help emerged as a much-welcomed response in Delaware. The program was backed by numerous public health and criminal justice stakeholders, including the County Executive, Attorney General and Delaware Department of Justice, and State Division of Substance Abuse and Mental Health. The program has four overarching goals:

- Provide direct (i.e., treatment) and indirect (i.e., housing, mental health, and transportation) supportive services to people suffering from a substance use disorder

Decrease the number of fatal and non-fatal overdoses

- Reduce contact with the criminal justice system associated with substance use as measured by arrests and incarceration

- Offer standing opportunities for immediate treatment among individuals who first ask for help and those who want to re-engage with the program

NCCPD connected program participants with a local treatment facility, where intake and clinical evaluations were completed to inform triage to the next level of care (Horn, 2016). The treatment facility used the 23-hour bed platform (aka social detox). Individuals who were not in active withdrawal or in need of a medical detox could then be admitted and supervised by medical professionals. Hero Help participants did not have to pay any of the program's costs. As Hero Help assisted with admissions to detox, program staff sought to enhance their roles in follow-up and re-engagement, as a lack of social supports and gaps in care can contribute to relapse (Streisel et al., 2019). The Hero Help program has since expanded its scope through two capacity development initiatives.

\section{CAPACITY DEVELOPMENT PHASE 1: THE HERO HELP COORDINATOR}

The Hero Help program first enhanced its activities via the University of Baltimore's Combating Opioid Overdoses through Community-Level Intervention (COOCLI) Initiative. Starting in December 2017, the program was able to provide 300 Narcan kits and corresponding training to people who survived overdoses and their loved ones. There was also a strong focus on expanding the program's outreach campaigns. The program was likewise able to hire a full-time civilian care coordinator based in NCCPD.

The Hero Help Coordinator manages all aspects of program engagement related to treatment, criminal justice involvement, and corresponding supportive services involving housing, employment, transportation, and mental health, among other needs. This person also plays a vital role in outreach, for example, by making attempts to subsequently contact people who experienced a non-fatal overdose and invite their participation in Hero Help. The coordinator effectively serves as a consistent point of contact for current participants and those who might benefit from joining the program in the future. This is in line with other police departments that have embedded full-time coordinators and clinicians in their agencies, evolving beyond the volunteer model of the Angel Initiative (e.g., see the Arlington, Massachusetts, Outreach Initiative in PAARI, 2021).

\section{Hero Help Participants, Program Activities, and Outcomes Under the Coordinator}

The first phase of capacity development also prompted an initial evaluation of the Hero Help program. A part-time research analyst affiliated with the Center for Drug and Health Studies (CDHS) at the University of Delaware and based in NCCPD gathered real-time and retrospective data on program participants and activities occurring between May 2016 and October 2018. This period reflects the initial implementation of the program (May 2016-February 2018) through the months following the hiring of the Hero Help coordinator (March 2018-October 2018) (Streisel et al., 2019). Information about participants and program outcomes was derived from case notes. Descriptive findings revealed who sought treatment via law enforcement as well as what activities can be undertaken to inform Delaware residents of this opportunity for recovery support.

Table I presents summary statistics associated with the characteristics of program participants. Among the 176 participants during this period, more than half identified as male $(65 \%)$. The median age of a Hero Help enrollee was 33 with a range from 18 to 67 years old. Most participants 
TABLE I Characteristics of Hero Help program participants $(n=176)$

\begin{tabular}{lc}
\hline \multicolumn{1}{c}{ Variable } & Mean \\
\hline Demographics & \\
Male (\%) & $65 \%$ \\
Female (\%) & $33 \%$ \\
Age (Mean) & 33 \\
White (\%) & $71 \%$ \\
Black (\%) & $6 \%$ \\
Other or missing & $23 \%$ \\
Drug use in last 30 days & \\
Any opiates (\%) & $86 \%$ \\
Heroin (\%) & $74 \%$ \\
Cocaine or crack cocaine (\%) & $46 \%$ \\
Marijuana (\%) & $32 \%$
\end{tabular}

identified as White (71\%), with $6 \%$ identifying as Black, and $23 \%$ having other or missing racial/ethnic identities. Relative to the general population of New Castle County communities outside of Wilmington (69\% White population and $21 \%$ Black population), White Delawareans were overrepresented in the program, but this pattern could be partly explained by disproportionate rates of opioid use (Cicero et al., 2014) and arrests for opioid-related possession offences (Donnelly et al., 2021) among White people. About $86 \%$ of participants reported using an opioid in the month preceding their enrolment, with nearly three-quarters stating they had used heroin during that time. Use of cocaine and marijuana were common, underscoring a need to treat the underlying issues that contribute to substance use in general, rather than opioid use alone.

The importance of having a civilian coordinator based in the agency cannot be minimized. Figure 1 summarizes program outcomes before and after the hiring of a Hero Help coordinator. Relative to the pre-coordinator period, detox program completion and acceptance of care after detox grew by $21 \%$ and $25 \%$, respectively. More participants remained engaged in the Hero Help Program at the end of the postcoordinator evaluation period. Arrests among participants also decreased by $23 \%$. These results suggest having a fulltime coordinator, who can get to know program participants and formulate individualized treatment plans, can greatly enhance program success.

Program activities also grew during this period. Partnerships with treatment providers increased by $50 \%(n=$ 27 ), offering more beds to new enrollees and options for re-engaged participants looking for a program to better fit their needs. Outreach among people who suffered a nonfatal overdose was challenging, but approximately $70 \%$ of individuals were reached by Hero Help staff through a home visit. Community outreach events $(n=28)$ were successful too, encouraging 56 people to seek treatment through Hero Help or another local program. As a result of these activities, the State of Delaware has identified the Hero Help program as a Community-Based Naloxone Access Program (Powell, 2019).

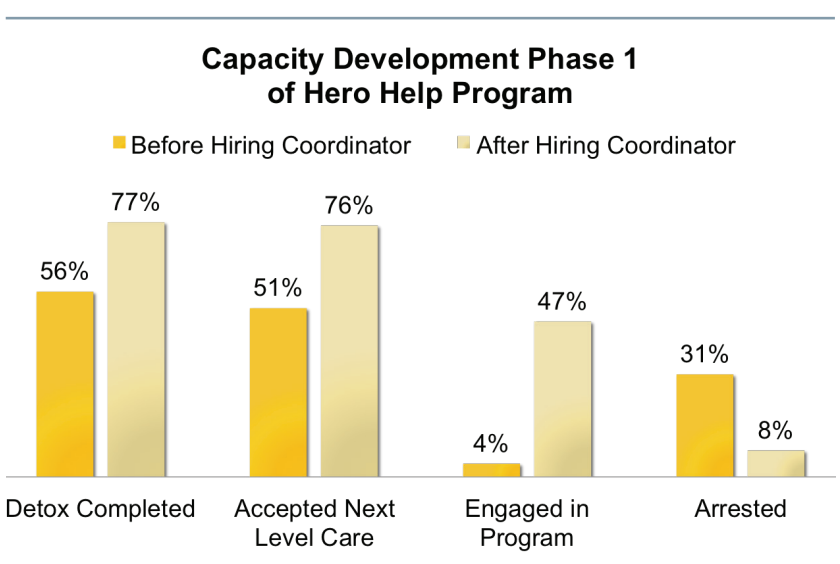

FIGURE 1 Program outcomes before and after hiring a Hero Help coordinator under the first phase of capacity development

\section{CAPACITY DEVELOPMENT PHASE 2: THE INFORM TEAM}

Within a year of hiring a program coordinator, the Hero Help program required additional support as the number of participants tripled. With the help of Bureau of Justice Assistance funding, NCCPD organized an Integrated NonFatal Overdose Response Mission (INFORM) team based in the NCCPD. The team still consists of the civilian program coordinator and police officers but now also includes a nurse, mental health professional (MHP), case manager, and child victim advocate. Of note, the MHP assists in identifying appropriate services for those with co-occurring substance use and mental health disorders. Meanwhile, the child victim advocate offers supportive services to children impacted by overdose. Training first responders on protocols when a child is exposed to substance misuse at home is also a priority. The INFORM team represents one part of NCCPD's Behavioral Health Unit tasked with assisting citizens presenting with substance misuse and mental health problems. Hero Help program participants $(n=544)$ and treatment providers $(n=52)$ have increased since 2018. A long-term evaluation is currently underway to assess the impacts of these program expansions.

\section{KEY LESSONS LEARNED}

Across these two periods of capacity development, Hero Help staff and affiliated officers shared several lessons learned. Three key takeaways involve enhancing credibility through collaboration, reducing stigma, and relying on data-driven approaches. Each lesson is explored with examples from the program below.

\section{Enhancing Credibility through Collaboration}

The implementation of this type of law enforcement-based treatment program often faces unique challenges because participants may be dually stigmatized by a substance use disorder and criminal justice involvement. NCCPD has relied on the following strategies to break down barriers and ensure successful partnerships among all members involved. 
- Rather than using media campaigns or excessive advertising to promote Hero Help, which was previously experimented with, NCCPD has instead focused on a bottom-up approach, operating through word of mouth within the recovery community. NCCPD partnered with organizations that work directly with people recovering from a substance use disorder to host events and promote the program. Having NCCPD members present at these events was an important component of relationship-building. NCCPD has likewise created a resource list of 39 agencies offering various supportive services.

- In conjunction with atTAck Addiction, a Delaware nonprofit organization (https://www.attackaddiction.org/), Hero Help formed People Empowering People (PEP) to help families navigate the process of recovery. People Empowering People provides awareness education, offers Narcan training, and hosts a speaker series to support all individuals who have a loved one undergoing the recovery process (see https://www.pepmembers.com/).

\section{Overcoming Stereotypes and Stigma}

The Hero Help program has also sought to address issues of stereotyping and stigma associated with substance use disorders and mental illness. While the following steps may seem simple or obvious, their importance should not be downplayed.

Officer rotation in outreach efforts has served as one way for Hero Help to reduce negative perceptions of individuals who have a substance use disorder and increase awareness about the capacity to assist. Officers are provided with talking points and resources, as officer responses to inquiries can determine whether an individual engages in or is turned off from the program. Direct involvement with recruitment has led to an increase in referrals among officers beyond the original target group of people who have overdosed.

- Hero Help also works to overcome stigma and emotional burnout by sharing success stories. Following up with officers on individuals they have recruited to the program helps officers to see the outcome of their work and stay motivated. The program highlights positive outcomes with treatment providers.

- Participants may worry about the stigma of engaging with police, especially if someone sees them being picked up in a police vehicle. One way Hero Help has attempted to alleviate this pressure is through the use of unmarked vehicles to transport participants who are entering the program.

- Hero Help has sought to identify and assist vulnerable populations. For instance, the program is collaborating with the Department of Justice to follow up on the needs of people involved with prostitution-related offences. Hero Help is working to create trust, demonstrate value, and remove blame from involvement in sex work due to an underlying substance use disorder. The goal is to have a conversation, not provide an ultimatum.

\section{Taking Data-Driven Approaches and Improving} Accessibility of Information

A key element of promoting the Hero Help program is collecting and providing data to document the program's growth and success. NCCPD has developed a case management system to track information concerning participants, outreach, and training. Specifically,

The department relies on REDCap, a secure web application where separate forms track participants, outreach, and training activities. Staff members have introduced additional forms to reflect changing program needs. For instance, a new form organizes referrals for children who might need help, even if their parents are not enrolled in the Hero Help program.

- Data-driven approaches allow NCCPD to examine equity in services and recruitment into the program. For example, participant data showed racial/ethnic disparities in who used the program. These disparities, in part, reflected missing data. Use of the REDCap system has reduced missing or other race/ethnicity information from $23 \%$ to $3 \%$ (currently $85 \%$ of participants identify as White and $12 \%$ as Black).

- NCCPD has partnered with the CDHS to analyze the program with each phase of capacity development. Data have made a difference in program implementation. A CDHS report demonstrated that program enrolment was largely following the demographics of overdose victims but indicated a need to enrol people from lower-income communities. Targeted Analytical Policing Strategies (TAPS) were used based on the data to provide monthly outreach by officers and members of the Delaware Division of Substance Abuse and Mental Health (DSAMH) to communities that may be overlooked because these areas have lower reporting rates and higher distrust of police presence.

- A CDHS report also demonstrated that approximately $60 \%$ of participants had experienced trauma. This finding led to the introduction of trauma-informed training and placing female participants with female case managers.

- The Hero Help program has also sought to reduce the difficulty of participation for providers and ensure that information is shared and disseminated in a clear and easily accessible format. NCCPD hosts regular meetings, distributes PowerPoint slides to providers so that they can share program materials on their websites, and is working on developing a stand-alone website for the Behavioral Health Unit.

\section{REMAINING CHALLENGES}

Hero Help continues to face challenges. The largest obstacle has been limited funding and resources among all organizations involved. Hero Help participants may come to detox with a wide range of needs (e.g., experience with trauma or human trafficking), and the detox provider may not be able to address all of them. Both sides can lose hope: the individual who shared their story and the provider who does not have the supports necessary to assist or is unable to connect that individual with appropriate care. A priority is to develop multidisciplinary care teams that can listen and have diverse resources necessary to assist those undergoing recovery. Another barrier involves communication challenges. There often is not a timely response when attempting to get 
ind ividuals into treatment for mental health. Bureaucratic justifications and obstacles can prevent individuals from getting admitted to the treatment they need. Lastly, Hero Help represents one programmatic response in a predominantly suburban jurisdiction. State-level efforts are being made to redress opioid-related problems in urban (e.g., Dover, DE, Morrison, 2017) and rural (e.g., Delaware Criminal Justice Council, 2019) communities. In addition, NCCPD is forging partnerships with other police departments in New Castle County to expand local opioid-related policy responses. Program expansion can help to reduce disparities in access to diversionary programs and the use of incarceration for drug-related offences (see Eichler, 2000; MacDonald \& Donnelly, 2016).

\section{CONCLUSION}

Rising levels of overdoses due to opioid misuse have fundamentally changed the role of law enforcement. Beyond serving as first responders to possible overdoses and receiving training in administering naloxone, some agencies like NCCPD have taken it upon themselves to connect people struggling with a substance use disorder to immediate treatment on a voluntary basis or to divert individuals out of the criminal justice system. The Hero Help program has evolved greatly in its first five years of implementation. While still connecting citizens with available beds in treatment facilities, it provides a holistic set of direct and indirect services with a full-time team of civilians and sworn officers based in the police department. To date, the program has worked with individuals, their families, and the broader community in initiating journeys to recovery. Hero Help is emerging as a promising model for public health and law enforcement partnerships aimed at redressing opioid misuse and increased overdoses in local communities.

\section{ACKNOWLEDGEMENTS}

We sincerely thank Colonel Vaughn Bond Jr., Ret. Colonel Elmer Setting, Captain John Treadwell Sr., Senior Lieutenant Allen Herring, Daniel Maas, Lori Kane, Officer Colleen Kearns, Officer Omari George and the rest of the Hero Help team for their efforts as well as their partnership with the University of Delaware's Center for Drug and Health Studies. We are also grateful for the research assistance provided by Laura Mutis. This project was supported by the University of Baltimore (PTE Federal Award grant number: G17799ONDCP06B), Delaware Department of Health and Social Services, Division of Public Health (Grant No. 19-395), and the U.S. Bureau of Justice Assistance (grant numbers: 2019-MO-BX-0013 and 2019-AR-BX-K027).

\section{CONFLICT OF INTEREST DISCLOSURES}

The authors declare that they have no conflicts of interest.

\section{AUTHOR AFFILIATIONS}

${ }^{*}$ Center for Drug and Health Studies, University of Delaware, Newark, DE, USA

\section{REFERENCES}

Anderson, T. L., Donnelly, E. A., Delcher, C., \& Wang, Y. (2021). Data science approaches in criminal justice and public health research: Lessons learned from opioid projects. Journal of Contemporary Criminal Justice, 37(2), 175-191. https://doi.org/10.1177/ 1043986221999858
Center for Disease Control Injury Center. (2020, March 19). Drug overdose deaths. https://www.cdc.gov/drugoverdose/data/statedeaths.html

Cicero, T. J., Ellis, M. S., Surratt, H. L., \& Kurtz, S. P. (2014). The changing face of heroin use in the United States: A retrospective analysis of the past 50 years. JAMA Psychiatry, 71(7), 821-826. https://doi. org/10.1001/jamapsychiatry.2014.366

Compton, W. M., Valentino, R. J., \& DuPont, R. L. (2021). Polysubstance use in the U.S. opioid crisis. Molecular Psychiatry, 26(1), 41-50. https://doi.org/10.1038/s41380-020-00949-3

Davis, C. S., Carr, D., Southwell, J. K., \& Beletsky, L. (2015). Engaging law enforcement in overdose reversal initiatives: Authorization and liability for naloxone administration. American Journal of Public Health, 105(8), 1530-1537. https://doi.org/10.2105/AJPH.2015.302638

Delaware Criminal Justice Council. (2019). Delaware Comprehensive Opioid Abuse Program (COAP) saving lives. Executive Office of the Governor of Delaware: Criminal Justice Council. https:// www.cossapresources.org/Content/Documents/GranteeProfiles/ Delaware_FY2019_Start.pdf

Donnelly, E. A., Wagner, J., Stenger, M., Cortina, H. G., O'Connell, D. J., \&Anderson, T. L. (2021). Opioids, race, and drug enforcement: Exploring local relationships between neighborhood context and Black-White opioid-related possession arrests. Criminal Justice Policy Review, 32(3): 219-244. https://doi.org/10.1177/ 0887403420911415

Eichler, T. (2000). Race and incarceration in Delaware: A preliminary consideration (pp. 1-28). Delaware Center for Justice and Metropolitan Wilmington Urban League. https://www.prisonpolicy.org/ scans/Racelncarceration.pdf

Formica, S. W., Apsler, R., Wilkins, L., Ruiz, S., Reilly, B., \& Walley, A. Y. (2018). Post opioid overdose outreach by public health and public safety agencies: Exploration of emerging programs in Massachusetts. International Journal of Drug Policy, 54, 43-50. https://doi. org/10.1016/i.drugpo.2018.01.001

Gloucester Police Department. (2015). Gloucester Police Department Volunteer ANGEL Program. Gloucester Police Department. http:// paariusa.org/wp-content/uploads/sites/46/2015/06/Angelprogram-policy-Aug-7-2015.pdf

Green, T. C., Zaller, N., Palacios, W. R., Bowman, S. E., Ray, M., Heimer, R., \& Case, P. (2013). Law enforcement attitudes toward overdose prevention and response. Drug and Alcohol Dependence, 133(2), 677-684. https://doi.org/10.1016/i.drugalcdep.2013.08.018

Hedegaard, H., Miniño, A. M., \&Warner, M. (2018). Drug overdose deaths in the United States, 1999-2017 (NCHS Data Brief No. 329). National Center for Health Statistics. https://www.cdc.gov/ nchs/products/databriefs/db329.htm

Horn, B. (2016, May 20). As Delaware heroin deaths rise, more treatment options sought. The News Journal. https://www.delawareonline. $\mathrm{com} /$ story/news/local/2016/05/20/delaware-heroin-deathscontinue-more-treatment-options-sought/82899870/

Jalal, H., Buchanich, J. M., Roberts, M. S., Balmert, L. C., Zhang, K., \& Burke, D. S. (2018). Changing dynamics of the drug overdose epidemic in the United States from 1979 through 2016. Science, 361(6408), eaaul 184. https://doi.org/10.1126/science.aaul184

Liu, S., Scholl, L., Hoots, B., \& Seth, P. (2020). Nonfatal drug and polydrug overdoses treated in emergency departments-29 States, 2018-2019. Morbidity and Mortality Weekly Report, 69(34), 1149-1155. https://doi.org/10.15585/mmwr.mm6934al

MacDonald, J. M., \& Donnelly, E. A. (2016). Evaluating the role of race in criminal justice adjudications in Delaware. Wilmington: Delaware Access to Justice Commission- Subcommittee on Fairness in the Adult Criminal Justice System. https://courts.delaware.gov/supreme/docs/ DE_DisparityReport.pdf 
Morrison, J. (2017). Dover's "ANGEL" initiative steers opioid users into treatment instead of jail. Retrieved July 10, 2021, from https://www. delawarepublic.org/post/dovers-angel-initiative-steers-opioid-userstreatment-instead-jail

PAARI. (2019). The Police Assisted Addiction \& Recovery Initiative (PAARI): About us. PAARI. https://paariusa.org/about-us/

PAARI. (2021). The Arlington Outreach Initiative. PAARI. https://paariusa. org/arlington/

Pearlman, J. (2017). Combatting Massachusetts's opioid epidemic: Reducing the social stigma of addiction through increased access to voluntary treatment services and expansion of mandatory clinician education programs. American Journal of Law \& Medicine. https:// doi.org/10.1177/0098858817701962

Powell, M. (2019, May 23). Hero Help. Addiction Policy Forum. https:// www.addictionpolicy.org/post/when-help-comes-calling

Purviance, D., Ray, B., Tracy, A., \& Southard, E. (2017). Law enforcement attitudes towards naloxone following opioid overdose training. Substance Abuse, 38(2), 177-182. https://doi.org/10.1080/08 897077.2016.1219439

Rando, J., Broering, D., Olson, J. E., Marco, C., \& Evans, S. B. (2015). Intranasal naloxone administration by police first responders is associated with decreased opioid overdose deaths. The American Journal of Emergency Medicine, 33(9), 1201-1204. https://doi. org/10.1016/i.ajem.2015.05.022
Samuels, M. (2016). Answer to opioid crisis: Treatment instead of jail. Boston University School of Public Health. https://www.bu.edu/ sph/news/articles/2016/looking-at-care-instead-of-jail-for-problemclose-to-home/

Stephenson, J. (2021). CDC warns of surge in drug overdose deaths during COVID-19. JAMA Health Forum, 2(1), e210001-e210001. https:// doi.org/10.1001/jamahealthforum.2021.0001

Streisel, S., O'Connell, D. J., Visher, C., \& Martin, S. S. (2019). Using law enforcement to improve treatment initiation and recovery. Federal Probation, 83(2), 39-44.

Vivolo-Kantor, A. M. (2018). Vital signs: Trends in emergency department visits for suspected opioid overdoses-United States, July 2016September 2017. MMWR. Morbidity and Mortality Weekly Report, 67. https://doi.org/10.15585/mmwr.mm6709el

Wagner, J., Neitzke-Spruill, L., O'Connell, D., Highberger, J., Martin, S. S., Walker, R., \& Anderson, T. L. (2019). Understanding geographic and neighborhood variations in overdose death rates. Journal of Community Health, 44(2), 272-283. https://doi.org/10.1007/ s10900-018-0583-0

Wagner, K. D., Liu, L., Davidson, P. J., Cuevas-Mota, J., Armenta, R. F., \& Garfein, R. S. (2015). Association between non-fatal opioid overdose and encounters with healthcare and criminal justice systems: Identifying opportunities for intervention. Drug and Alcohol Dependence, 153, 215-220. https://doi.org/10.1016/i.drugalcdep.2015.05.026 